\title{
Nonlinear Zeeman Splitting of Holes in Doped GaAs Heterostructures
}

\author{
M. Kubisa ${ }^{a}$, K. Ryczko ${ }^{a}$, J. JAdczAK ${ }^{a}$, L. Bryja ${ }^{a}$, J. Misiewicz ${ }^{a}$ And M. Potemski ${ }^{b}$ \\ ${ }^{a}$ Institute of Physics, Wrocław University of Technology, Wybrzeże Wyspiańskiego 27, 50-370 Wrocław, Poland \\ ${ }^{b}$ Grenoble High Magnetic Field Laboratory, CNRS, Grenoble, France
}

\begin{abstract}
Polarization-resolved photoluminescence from two-dimensional GaAs/GaAlAs heterostructures doped with acceptors was studied in high magnetic fields. Measurements were carried out in low temperatures up to $2 \mathrm{~K}$ and magnetic field up to $21 \mathrm{~T}$. Experiments performed in the Faraday configuration enabled to resolve hole states with different spin orientation. We observed a nonlinear behavior of valence-band $g$ factor in strong magnetic fields. To explain obtained results, a detailed theoretical calculation was carried out based on the Luttinger model for valence-band states. We examined the spin splitting of hole levels under the influence of both external magnetic field and built-in electric field existing in doped heterostructures. Changes of hole $g$ factor with the width of the structure and the density of two-dimensional carriers are discussed.
\end{abstract}

PACS: $75.75 .-\mathrm{C}$

\section{Introduction}

The effective $g$-factor of electrons, holes, and excitons in semiconductor systems with reduced dimensionality has recently received considerable attention since it provides detailed information on the subband structure. Due to the spin-orbit interaction the Landé factor in solids may deviate strongly from the free-electron $g_{0}=2.0023$ value and can be as large as -51 in the narrow-gap material InSb. Strong electric fields present in low-dimensional structures further modify the spin splitting and $g$ factor becomes dependent on the sample geometry and doping profile. The interest in spin properties of semiconductor heterostructures was increased with the invention of spintronics offering radical alternatives to conventional electronics [1]. Most of the theoretical and experimental investigations in this field were devoted to the studies of electron $g$ factor, spin properties of confined holes have received less attention. As a result of low symmetry, the heavy-hole $g$ factor was shown to exhibit a strong anisotropy with a significant difference between the components in the direction of, and perpendicular to the plane of the quantum well [2]. The longitudinal component in GaAs/GaAlAs heterostructures was determined experimentally as a function of the well width from the Zeeman splitting of lines luminescence [3]. Low-field measurements of Traynor et al. [4] revealed a highly nonlinear nature of hole spin splitting in wide quantum wells. Some experimental studies were performed in strained InGaAs/GaAs systems under high magnetic fields [5]. The aim of this paper was to study both experimentally and theoretically the influence of a strong magnetic field on the longitudinal $g$ factor of heavy holes in GaAs/ GaAlAs heterostructures.
The photoluminescence measurements were performed for a variety of $p$-type GaAs/GaAlAs quantum wells with different widths, doping levels and mobilities. The results presented here concern a $22 \mathrm{~nm}$ wide GaAs/ $\mathrm{Ga}_{0.65} \mathrm{Al}_{0.35} \mathrm{As}$ quantum well, grown by molecular beam epitaxy on a (001) semi-insulating GaAs substrate and $\delta$-carbon doped in the barrier on one side. The well was separated from the doping layer by a $40 \mathrm{~nm}$ spacer. The hole mobility measured at $T=4.2 \mathrm{~K}$ was $\mu=$ $1.71 \times 10^{5} \mathrm{~cm}^{2} /(\mathrm{V} \mathrm{s})$. The quantum Hall effect measurements in the van der Pauw configuration at $T \leq 4.2 \mathrm{~K}$ gave the temperature independent hole concentration $N_{\mathrm{s}}=1.43 \times 10^{11} \mathrm{~cm}^{-2}$ (in the dark). Under the laser illumination $N_{\mathrm{s}}$ decreased up to several percents, linearly in the excitation-power density. The photoluminescence was excited by the $532.8 \mathrm{~nm}$ line of a helium-neon laser, above the band gap of the barrier. The optical experiments were performed in the Faraday configuration at $T=2.3 \mathrm{~K}$, using magnetic fields up to $B=21 \mathrm{~T}$ varied by a small step $\Delta B=0.05 \mathrm{~T}$. The switch between $\sigma^{-}$ and $\sigma^{+}$helices was achieved by reversing the direction of field. To analyze the spectra we used a $0.55 \mathrm{~m}$ long monochromator, with a nitrogen-cooled 2024-pixel CCD camera.

\section{Results and discussion}

In the $B=0$ photoluminescence spectrum a single line was observed for all used excitation-power densities. The magnetic field applied along the growth direction split this line into three components in both $\sigma^{-}$and $\sigma^{+}$polarizations as shown in Fig. 1. At magnetic fields above $6 \mathrm{~T}$ we could resolve the energy position of all lines. A detailed analysis of experimental data and a comparison 
with numerical calculations of the structure and our previous studies [6] allowed to attribute the observed lines to neutral exciton $(\mathrm{X})$ and positively charged exciton in singlet $\left(\mathrm{X}_{\mathrm{s}}^{+}\right)$and dark triplet $\left(\mathrm{X}_{\mathrm{td}}^{+}\right)$states. The excitonic Landé factor was determined from the evolution of energy position of the neutral exciton.

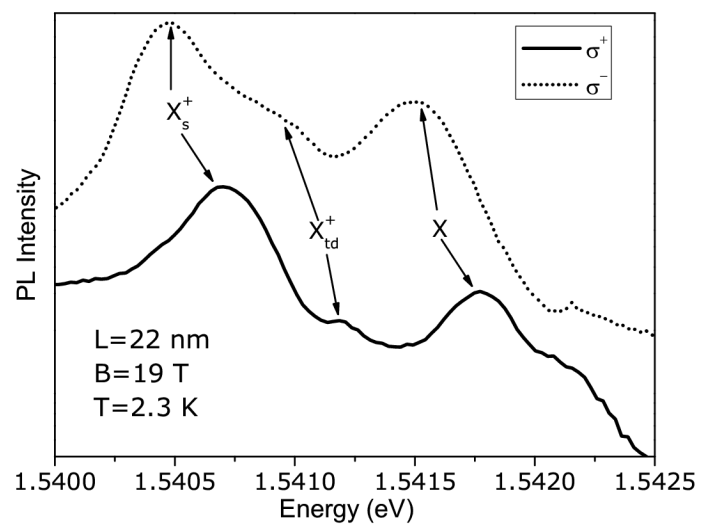

Fig. 1. Photoluminescence spectra of a $22 \mathrm{~nm}$ wide GaAs $/ \mathrm{Ga}_{0.65} \mathrm{Al}_{0.35}$ As quantum well measured at $B=$ $19 \mathrm{~T}$ in the Faraday configuration. The lines denote as $\mathrm{X}, \mathrm{X}_{\mathrm{td}}^{+}$and $\mathrm{X}_{\mathrm{s}}^{+}$are attributed to neutral and positively charged (triplet and singlet) excitonic states, respectively.

In theoretical calculations the Luttinger and Kohn model [7] was used to describe the valence-band states in studied heterostructures. The influence of strong magnetic field directed parallel to the growth axis was included in the axial approximation [8]. To calculate the energies and wave functions of confined holes we applied the numerical procedure developed in Ref. [9]. The potential profiles and subband energies in doped quantum wells were determined in a self-consistent way, taking into account all known structure parameters. We assumed that the MBE-grown GaAs layer is of $p$-type, with background doping of $5 \times 10^{14} \mathrm{~cm}^{-3}$. In the calculations the standard set of Luttinger parameters was used: $\gamma_{1}=6.85, \gamma_{2}=2.1, \gamma_{3}=2.9, \kappa=1.2$ for GaAs and $\gamma_{1}=3.45, \gamma_{2}=0.68, \gamma_{3}=1.29, \kappa=0.12$ for AlAs.

Figure 2 shows calculated hole energies in a $22 \mathrm{~nm}$ quantum well with the free carrier density $N_{\mathrm{s}}=1.8 \times$ $10^{11} \mathrm{~cm}^{-2}$. We plotted merely the positions of the Landau levels with the highest energies in the first valence subband. They display strongly nonlinear behavior resulting from the valence band mixing. As the magnetic field is increased the hole states are linearly shifted and split by the diagonal elements of the Luttinger matrix, while the off-diagonal terms lead to level repulsion and in effect to the nonlinearity. Inset presents the computed valence-band profile and the squared wave function at the subband edge for $B=0$.

In order to evaluate the spin splitting of valence states the exact wave functions of hole Landau levels were used to calculate the mean values of the Hamiltonian

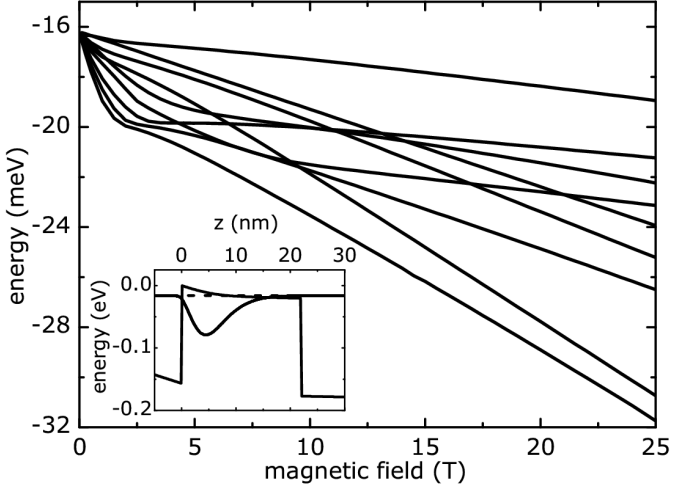

Fig. 2. Landau levels in the first heavy-hole subband calculated for the $22 \mathrm{~nm}$ quantum well with the carrier density $N_{\mathrm{s}}=1.8 \times 10^{11} \mathrm{~cm}^{-2}$. Inset shows the valence-band profile and the squared wave function at the subband edge for $B=0$.

$H_{z}=2 \mu_{\mathrm{B}} \kappa B J_{z}$. Here $\mu_{\mathrm{B}}$ is the Bohr magneton, $J_{z}$ is the Luttinger matrix and $H_{z}$ represents the part of Luttinger Hamiltonian responsible for the Zeeman splitting. As was shown in Ref. [9], the strongest interband optical transitions in the Faraday configuration involve valence-band Landau levels $n=1$ (in the $\sigma^{-}$polarization) and $n=-2$ $\left(\sigma^{+}\right)$. The hole $g$ factor was calculated from the difference of mean $H_{z}$ values in these states. The results plotted as a function of magnetic field intensity are shown in Fig. 3. Overall the hole $g$ factor increases abruptly with $B$ in the low-field regime and at some value of $B$, which depends on the structure size and doping, becomes saturated. Part (a) presents $g_{\mathrm{h}}$ calculated for quantum wells with equal doping and hole density $N_{\mathrm{s}}=1.8 \times 10^{11} \mathrm{~cm}^{-2}$, but of different width $L$. The spin splitting of hole states is sensitive to the size of structure in the region of small $g_{\mathrm{h}}$ magnetic fields and increases as $L$. At higher fields $g_{\mathrm{h}}$ reaches the value close to 1.1 , almost independent of the width of well. In part (b) we show the $g$ factor calculated for $22 \mathrm{~nm}$ structures with different doping. As the density $N_{\mathrm{s}}$ of holes is increased the spin splitting becomes reduced. This can be explained as an outcome of the growing built-in electric field which rotates the hole spin towards the well plane [2].

The points in Fig. 3 represent the experimental data. As the Zeeman splitting of the heavy-hole-electron excitonic emission is characterized by $g_{\mathrm{ex}}=g_{\mathrm{e}}+g_{\mathrm{h}}[10]$, the hole $g$ factor was determined by substracting $g_{\mathrm{e}}$ from the measured $g_{\text {ex }}$ values. It was show [3] that in wide quantum wells with $L>15 \mathrm{~nm}$ the electron Landé factor approaches the bulk GaAs value $g_{\mathrm{e}}=-0.44$. The measured hole $g$ factor agrees reasonably well with the results of calculations, especially in the low-field regime, where $g_{\mathrm{h}}$ increases rapidly with $B$. At the highest fields used in the experiment $g_{\mathrm{h}}$ tends to saturate, however both the saturation field and saturated value clearly exceed the values predicted by theory. This is likely due to the fact that in wide heterostructures the separation between the 


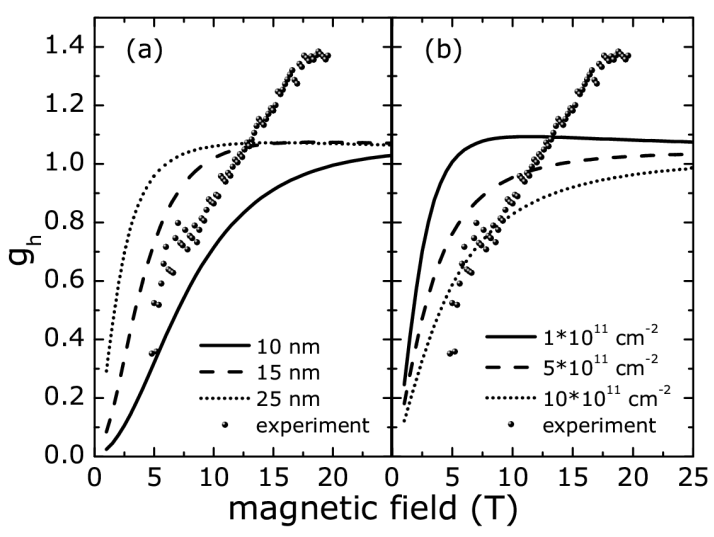

Fig. 3. Magnetic field dependence of calculated (lines) and measured (points) heavy hole $g$ factor. Experimental data were obtained for the $22 \mathrm{~nm}$ quantum well by substracting $g_{\mathrm{e}}=-0.44$ from the measured excitonic $g$ factor. Part (a) shows theoretical results for quantum wells with equal hole density $N_{\mathrm{s}}=1.8 \times 10^{11} \mathrm{~cm}^{-2}$ and different widths. Part (b) presents $g$ factor calculated for $22 \mathrm{~nm}$ wells with different doping.

light and heavy-hole subbands becomes comparable to the excitonic binding energy. Therefore the Coulomb interaction mixes the hole states from different subbands with very different $g$ factors. The excitonic spin splitting in wide heterostructures is not expected to measure the spin properties of heavy-hole states $[4,11]$.

\section{References}

[1] I. Žutić, J. Fabian, S. Das Sarma, Rev. Mod. Phys. 76, 323 (2004).

[2] R. Winkler, Spin-Orbit Coupling Effects in Two-Dimensional Electron and Hole Systems, Springer, Berlin 2003.

[3] M.J. Snelling, E. Blackwood, C.J. McDonagh, R.T. Harley, C.T.B. Foxon, Phys. Rev. B 45, 3922 (1992).

[4] N.J. Traynor, R.J. Warburton, M.J. Snelling, R.T. Harley, Phys. Rev. B 55, 15701 (1997).

[5] Th. Wimbauer, K. Oettinger, Al. L. Efros, B.K. Meyer, H. Brugger, Phys. Rev. B 50, 8889 (1994), R.J. Warburton, R.J. Nicholas, S. Sasaki, N. Miura, K. Woodbridge, Phys. Rev. B 48, 12323 (1993).

[6] L. Bryja, A. Wójs, J. Misiewicz, M. Potemski, D. Reuter, A. Wieck, Phys. Rev. B 75, 035308 (2007).

[7] J.M. Luttinger, W. Kohn, Phys. Rev. 97, 869 (1953).

[8] S.-R. Eric Yang, D.A. Broido, L.J. Sham, Phys. Rev. B 32, 6630 (1980).

[9] M. Kubisa, L. Bryja, K. Ryczko, J. Misiewicz, C. Bardot, M. Potemski, G. Ortner, M. Bayer, A. Forchel, C.B. Sorensen, Phys. Rev. B 67, 035305 (2003).

[10] H.W. van Kesteren, E.C. Cosman, W.A.J.A. van der Poel, C.T. Foxon, Phys. Rev. B 41, 5283 (1990).

[11] G.E.W. Bauer, T. Ando, Phys. Rev. B 37, 3130 (1988). 